\title{
Learning with Randomized Majority Votes
}

\author{
Alexandre Lacasse, François Laviolette, \\ Mario Marchand, and Francis Turgeon-Boutin \\ Department of Computer Science and Software Engineering, \\ Laval University, Québec (QC), Canada
}

\begin{abstract}
We propose algorithms for producing weighted majority votes that learn by probing the empirical risk of a randomized (uniformly weighted) majority vote - instead of probing the zero-one loss, at some margin level, of the deterministic weighted majority vote as it is often proposed. The learning algorithms minimize a risk bound which is convex in the weights. Our numerical results indicate that learners producing a weighted majority vote based on the empirical risk of the randomized majority vote at some finite margin have no significant advantage over learners that achieve this same task based on the empirical risk at zero margin. We also find that it is sufficient for learners to minimize only the empirical risk of the randomized majority vote at a fixed number of voters without considering explicitly the entropy of the distribution of voters. Finally, our extensive numerical results indicate that the proposed learning algorithms are producing weighted majority votes that generally compare favorably to those produced by AdaBoost.
\end{abstract}

\section{Introduction}

Randomized majority votes (RMVs) were proposed by [9] as a theoretical tool to provide a margin-based risk bound for weighted majority votes such as those produced by AdaBoost 2]. Given a distribution $Q$ over a (possibly continuous) space $\mathcal{H}$ of classifiers, a RMV is a uniformly weighted majority vote of $N$ classifiers where each classifier is drawn independently at random according to $Q$. For infinitely large $N$, the RMV becomes identical to the $Q$-weighted majority vote over $\mathcal{H}$. The RMV is an example of a stochastic classifier having a risk (i.e., a generalization error) that can be tightly upper bounded by a PAC-Bayes bound. Consequently, 6] have used the PAC-Bayes risk bound of [8] (see also [7]) to obtain a tighter margin-based risk bound than the one proposed by 9 . Both of these bounds depend on the empirical risk, at some margin level $\theta$, made by the $Q$-weighted majority vote and on some regularizer. In the case of [9], the regularizer depends on the cardinality of $\mathcal{H}$ (or its $\mathrm{VC}$ dimension in the case of a continuous set) and, consequently, the only learning principle that can be inferred from this bound is to choose $Q$ in order to maximize the margin. In the case of [6], the regularizer depends on the Kullback-Leibler divergence $\mathrm{KL}(Q \| P)$ between a prior distribution $P$ and the posterior $Q$. Consequently, when $P$ is uniform, the design principle inferred from this bound is to choose $Q$ to maximize 
both the margin and the entropy. We emphasize that both of these risk bounds are NP-hard to minimize because they depend on the empirical risk at margin $\theta$ of the $Q$-weighted majority vote as measured by the zero-one loss. Maximum entropy discrimination [4] is a computationally feasible method to maximize the entropy while maintaining a large margin by using classification constraints on each training examples. This is done by incorporating a prior on margin values for each training example which yield slack variables similar as those used for the SVM. These classification constraints are introduced, however, in an ad-hoc way which does not follow from a risk bound.

In this paper, we propose to use PAC-Bayes bounds for the risk of the $Q$ weighted majority vote, tighter than the one proposed by [6], which depend on the empirical risk of the randomized (uniformly weighted) majority voteinstead of depending on the empirical risk of the deterministic $Q$-weighted majority vote. As we shall see, the risk of the randomized majority vote (RMV) on a single example is non convex. But it can be tightly upper-bounded by a convex surrogate - thus giving risk bounds which are convex in $Q$ and computationally cheap to minimize. We therefore propose learning algorithms that minimize these convex risk bounds. These are algorithms that basically learn by finding a distribution $Q$ over $\mathcal{H}$ having large entropy and small empirical risk for the associated randomized majority vote. Hence, instead of learning by probing the empirical risk of the deterministic majority vote (as suggested by [9] and [6]), we propose to learn by probing the empirical risk of the randomized (uniformly weighted) majority vote. Our approach thus differs substantially from maximum entropy discrimination [4] where the empirical risk of the RMV is not considered.

Recently, 3. have also proposed learning algorithms that construct a weighted majority vote by minimizing a PAC-Bayes risk bound. However, their approach was restricted to the case of isotropic Gaussian posteriors over the set of linear classifiers. In this paper, both the posterior $Q$ and the set $\mathcal{H}$ of basis functions are completely arbitrary. However, the algorithms that we present here apply only to the case where $\mathcal{H}$ is finite.

Our numerical results indicate that learners producing a weighted majority vote based on the empirical risk of the randomized majority vote at some finite margin $\theta$ have no significant advantage over learners that achieve this same task based on the empirical risk at zero margin. Perhaps surprisingly, we also find that it is sufficient for learners to minimize only the empirical risk of the randomized majority vote at a fixed number of voters without considering explicitly the entropy of the distribution of voters. Finally, our extensive numerical results indicate that the proposed learning algorithms are producing weighted majority votes that generally compare favorably to those produced by AdaBoost.

\section{Definitions and PAC-Bayes Theory}

We consider the binary classification problem where each example is a pair $(\mathbf{x}, y)$ such that $y \in\{-1,+1\}$ and $\mathbf{x}$ belongs to an arbitrary set $\mathcal{X}$. As usual, we assume that each example $(\mathbf{x}, y)$ is drawn independently according to a fixed, but unknown, distribution $D$ on $\mathcal{X} \times\{-1,+1\}$. 
The risk $R(h)$ of any classifier $h$ is defined as the probability that $h$ misclassifies an example drawn according to $D$. Given a training set $S=\left\{\left(\mathbf{x}_{i}, y_{i}\right)\right.$, $\left.\ldots,\left(\mathbf{x}_{m}, y_{m}\right)\right\}$ of $m$ examples, the empirical risk $R_{S}(h)$ of $h$ is defined by its frequency of training errors on $S$. Hence,

$$
R(h) \stackrel{\text { def }}{=} \underset{(\mathbf{x}, y) \sim D}{\mathbf{E}} I(h(\mathbf{x}) \neq y) \quad ; \quad R_{S}(h) \stackrel{\text { def }}{=} \frac{1}{m} \sum_{i=1}^{m} I\left(h\left(\mathbf{x}_{i}\right) \neq y_{i}\right),
$$

where $I(a)=1$ if predicate $a$ is true and 0 otherwise.

The so-called PAC-Bayes theorems [107/53] provide guarantees for a stochastic classifier called the Gibbs classifier. Given a distribution $Q$ over a (possibly continuous) space $\mathcal{H}$ of classifiers, the Gibbs classifier $G_{Q}$ is defined in the following way. Given an input example $\mathbf{x}$ to classify, $G_{Q}$ chooses randomly a (deterministic) classifier $h$ according to $Q$ and then classifies $\mathbf{x}$ according to $h(\mathbf{x})$. The risk $R\left(G_{Q}\right)$ and the empirical risk $R_{S}\left(G_{Q}\right)$ of the Gibbs classifier are thus given by

$$
R\left(G_{Q}\right)=\underset{h \sim Q}{\mathbf{E}} R(h) \quad ; \quad R_{S}\left(G_{Q}\right)=\underset{h \sim Q}{\mathbf{E}} R_{S}(h) .
$$

To upper bound $R\left(G_{Q}\right)$, we will make use of the following PAC-Bayes theorem due to [1]; see also [3]. In contrast, [6] used the looser bound of [8].

Theorem 1. For any distribution $D$, any set $\mathcal{H}$ of classifiers, any distribution $P$ of support $\mathcal{H}$, any $\delta \in(0,1]$, and any positive real number $C$, we have

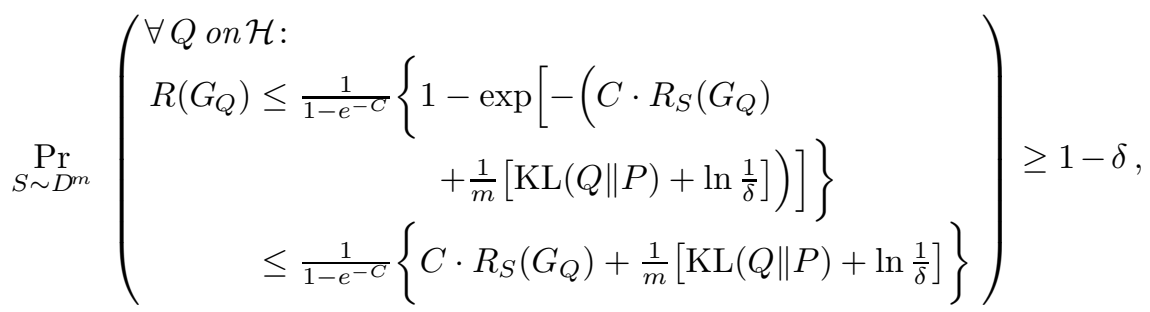

where $\mathrm{KL}(Q \| P) \stackrel{\text { def }}{=} \underset{h \sim Q}{\mathbf{E}} \ln \frac{Q(h)}{P(h)}$ is the Kullback-Leibler divergence between $Q$ and $P$.

The second inequality, obtained by using $1-e^{-x} \leq x$, gives a looser bound which is, however, easier to interpret.

In Theorem 11 the prior distribution $P$ must be defined a priori without reference to the training data $S$. Hence, $P$ cannot depend on $S$ whereas arbitrary dependence on $S$ is allowed for the posterior $Q$. Finally note that the bound of Theorem 1 holds for any constant $C$. Thanks to the standard union bound argument, the bound can be made valid uniformly for $k$ different values of $C$ by replacing $\delta$ with $\delta / k$.

\section{Specialization to Randomized Majority Votes}

Given a distribution $Q$ over a space $\mathcal{H}$ of classifiers, we are often more interested in predicting according to the deterministic weighted majority vote $B_{Q}$ instead 
of the stochastic Gibbs classifier $G_{Q}$. On any input example $\mathbf{x}$, the output $B_{Q}(\mathbf{x})$ of $B_{Q}$ is given by

$$
B_{Q}(\mathbf{x}) \stackrel{\text { def }}{=} \operatorname{sgn}[\underset{h \sim Q}{\mathbf{E}} h(\mathbf{x})],
$$

where $\operatorname{sgn}(s)=+1$ if $s>0$ and -1 otherwise.

Theorem 11. however, provides a guarantee for $R\left(G_{Q}\right)$ and not for the risk $R\left(B_{Q}\right)$ of the weighted majority vote $B_{Q}$. As an attempt to characterize the quality of weighted majority votes, let us analyze a special type of Gibbs classifier, closely related to $B_{Q}$, that we call the randomized majority vote (RMV).

Given a distribution $Q$ over $\mathcal{H}$ and a natural number $N$, the randomized majority vote $G_{Q^{N}}$ is a uniformly weighted majority vote of $N$ classifiers chosen independently at random according to $Q$. Hence, to classify $\mathbf{x}, G_{Q^{N}}$ draws $N$ classifiers $\left\{h_{k(1)}, \ldots, h_{k(N)}\right\}$ from $\mathcal{H}$ independently according to $Q$ and classifies $\mathbf{x}$ according to $\operatorname{sgn}(g(\mathbf{x}))$, where

$$
g(\mathbf{x}) \stackrel{\text { def }}{=} \frac{1}{N} \sum_{i=1}^{N} h_{k(i)}(\mathbf{x}) .
$$

We denote by $g \sim Q^{N}$, the above-described process of choosing $N$ classifiers according to $Q^{N}$ to form $g$.

Let us denote by $W_{Q}(\mathbf{x}, y)$ the fraction of classifiers, under measure $Q$, that misclassify example $(\mathbf{x}, y)$ :

$$
W_{Q}(\mathbf{x}, y) \stackrel{\text { def }}{=} \underset{h \sim Q}{\mathbf{E}} I(h(\mathbf{x}) \neq y) .
$$

For simplicity, let us limit ourselves to the case where $N$ is odd. In that case, $g(\mathbf{x}) \neq 0 \forall \mathbf{x} \in \mathcal{X}$.

Similarly, denote by $W_{Q^{N}}(\mathbf{x}, y)$ the fraction of uniformly weighted majority votes of $N$ classifiers, under measure $Q^{N}$, that err on $(\mathbf{x}, y)$ :

$$
\begin{aligned}
W_{Q^{N}}(\mathbf{x}, y) & \stackrel{\text { def }}{=} \underset{g \sim Q^{N}}{\mathbf{E}} I(\operatorname{sgn}[g(\mathbf{x})] \neq y) \\
& =\underset{g \sim Q^{N}}{\mathbf{E}} I(y g(\mathbf{x})<0) \\
& =\underset{g \sim Q^{N}}{\operatorname{Pr}}(y g(\mathbf{x})<0) .
\end{aligned}
$$

Recall that $W_{Q}(\mathbf{x}, y)$ is the probability that a classifier $h \in \mathcal{H}$, drawn according to $Q$, err on $\mathbf{x}$. Since $y g(\mathbf{x})<0$ iff more than half of the classifiers drawn according to $Q$ err on $\mathbf{x}$, we have

$$
W_{Q^{N}}(\mathbf{x}, y)=\sum_{k=\left\lceil\frac{N}{2}\right\rceil}^{N}\left(\begin{array}{l}
N \\
k
\end{array}\right) W_{Q}^{k}(\mathbf{x}, y)\left[1-W_{Q}(\mathbf{x}, y)\right]^{N-k} .
$$

Note that, with these definitions, the risk $R\left(G_{Q^{N}}\right)$ of the randomized majority vote $G_{Q^{N}}$ and its empirical estimate $R_{S}\left(G_{Q^{N}}\right)$ on a training set $S$ of $m$ examples are respectively given by 


$$
R\left(G_{Q^{N}}\right)=\underset{(\mathbf{x}, y) \sim D}{\mathbf{E}} W_{Q^{N}}(\mathbf{x}, y) \quad ; \quad R_{S}\left(G_{Q^{N}}\right)=\frac{1}{m} \sum_{i=1}^{m} W_{Q^{N}}\left(\mathbf{x}_{i}, y_{i}\right) .
$$

Since the randomized majority vote $G_{Q^{N}}$ is a Gibbs classifier with a distribution $Q^{N}$ over the set of all uniformly weighted majority votes that can be realized with $N$ base classifiers chosen from $\mathcal{H}$, we can apply to $G_{Q^{N}}$ the PAC-Bayes bound given by Theorem 1. To achieve this specialization, we only need to replace $Q$ and $P$ by $Q^{N}$ and $P^{N}$ respectively and use the fact that

$$
\mathrm{KL}\left(Q^{N} \| P^{N}\right)=N \cdot \mathrm{KL}(Q \| P) .
$$

Consequently, given this definition for $G_{Q^{N}}$, Theorem 1 admits the following corollary.

Corollary 1. For any distribution $D$, any set $\mathcal{H}$ of base classifiers, any distribution $P$ of support $\mathcal{H}$, any $\delta \in(0,1]$, any positive real number $C$, and any non-zero positive integer $N$, we have

$$
\operatorname{Pr}_{S \sim D^{m}}\left(\begin{array}{rl}
\forall Q \text { on } \mathcal{H}: & \\
R\left(G_{Q^{N}}\right) \leq \frac{1}{1-e^{-C}} & \left\{1-\exp \left[-\left(C \cdot R_{S}\left(G_{Q^{N}}\right)\right.\right.\right. \\
& \left.\left.\left.+\frac{1}{m}\left[N \cdot \mathrm{KL}(Q \| P)+\ln \frac{1}{\delta}\right]\right)\right]\right\}
\end{array}\right) \geq 1-\delta .
$$

By the standard union bound argument, the above corollary will hold uniformly for $k$ values of $C$ and all $N>1$ if we replace $\delta$ by $\frac{6 \delta}{k(N \pi)^{2}}$ (in view of the fact that $\left.\sum_{i=1}^{\infty} i^{-2}=\pi^{2} / 6\right)$. Figure 1 shows the behavior of $W_{Q^{N}}(\mathbf{x}, y)$ as a function of $W_{Q}(\mathbf{x}, y)$ for different values of $N$. We can see that $W_{Q^{N}}(\mathbf{x}, y)$ tends to the
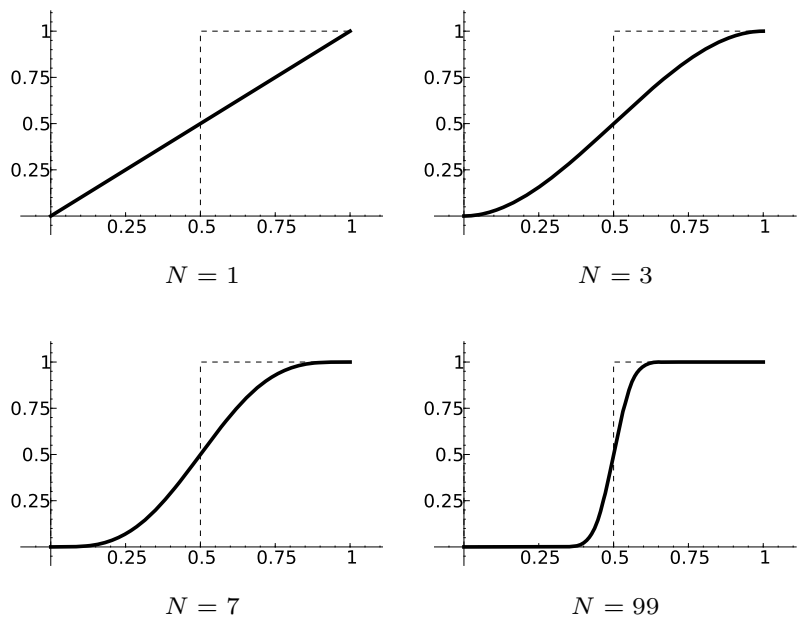

Fig. 1. Plots of $W_{Q^{N}}(\mathbf{x}, y)$ as a function of $W_{Q}(\mathbf{x}, y)$ for different values of $N$ 
zero-one loss $I\left(W_{Q}(\mathbf{x}, y)>1 / 2\right)$ of the weighted majority vote $B_{Q}$ as $N$ is increased.

Since $W_{Q^{N}}(\mathbf{x}, y)$ is monotone increasing in $W_{Q}(\mathbf{x}, y)$ and $W_{Q^{N}}(\mathbf{x}, y)=1 / 2$ when $W_{Q}(\mathbf{x}, y)=1 / 2$, it immediately follows that $I\left(W_{Q}(\mathbf{x}, y)>1 / 2\right) \leq$ $2 W_{Q^{N}}(\mathbf{x}, y)$ for all $N$ and $(\mathbf{x}, y)$. Consequently $R\left(B_{Q}\right) \leq 2 R\left(G_{Q^{N}}\right)$ and Corollary 1 provides an upper bound to $R\left(B_{Q}\right)$ via this "factor of two" rule.

\section{Margin Bound for the Weighted Majority Vote}

Since the risk of the weighted majority vote can be substantially smaller that Gibbs' risk, it may seem too crude to upper bound $R\left(B_{Q}\right)$ by $2 R\left(G_{Q^{N}}\right)$. One way to get rid of this factor of two is to consider the relation between $R\left(B_{Q}\right)$ and Gibbs' risk $R^{\theta}\left(G_{Q^{N}}\right)$ at some positive margin $\theta$. [9] have shown that

$$
R\left(B_{Q}\right) \leq R^{\theta}\left(G_{Q^{N}}\right)+e^{-N \theta^{2} / 2},
$$

where

$$
R^{\theta}\left(G_{Q^{N}}\right) \stackrel{\text { def }}{=} \underset{(\mathbf{x}, y) \sim D}{\mathbf{E}} \underset{g \sim Q^{N}}{\operatorname{Pr}}(y g(\mathbf{x}) \leq \theta) .
$$

Hence, for sufficiently large $N \theta^{2}$, Equation 1 provides an improvement over the "factor of two rule" as long as $R^{\theta}\left(G_{Q^{N}}\right)$ is less than $2 R\left(G_{Q^{N}}\right)$.

Following this definition of $R^{\theta}\left(G_{Q^{N}}\right)$, let us denote by $W_{Q^{N}}^{\theta}(\mathbf{x}, y)$ the fraction of uniformly weighted majority votes of $N$ classifiers, under measure $Q^{N}$, that err on $(\mathbf{x}, y)$ at some margin $\theta$, i.e.,

$$
W_{Q^{N}}^{\theta}(\mathbf{x}, y) \stackrel{\text { def }}{=} \underset{g \sim Q^{N}}{\operatorname{Pr}}(y g(\mathbf{x}) \leq \theta) .
$$

Consequently,

$$
R^{\theta}\left(G_{Q^{N}}\right)=\underset{(\mathbf{x}, y) \sim D}{\mathbf{E}} W_{Q^{N}}^{\theta}(\mathbf{x}, y) \quad ; \quad R_{S}^{\theta}\left(G_{Q^{N}}\right)=\frac{1}{m} \sum_{i=1}^{m} W_{Q^{N}}^{\theta}\left(\mathbf{x}_{i}, y_{i}\right) .
$$

For $N$ odd, $N y g(\mathbf{x})$ can take values only in $\{-N,-N-2, \ldots,-1,+1, \ldots,+N\}$. We can thus assume, without loss of generality (w.l.o.g.), that $\theta$ can only take $(N+1) / 2+1$ values.

To establish the relation between $W_{Q^{N}}^{\theta}(\mathbf{x}, y)$ and $W_{Q}(\mathbf{x}, y)$, note that $y g(\mathbf{x}) \leq$ $\theta$ iff

$$
1-\frac{2}{N} \sum_{i=1}^{N} I\left(h_{k(i)}(\mathbf{x}) \neq y\right) \leq \theta .
$$

The randomized majority vote $G_{Q^{N}}$ thus misclassifies $(\mathbf{x}, y)$ at margin $\theta$ iff at least $\left\lceil\frac{N}{2}(1-\theta)\right\rceil$ of its voters err on $(\mathbf{x}, y)$. Consequently,

$$
W_{Q^{N}}^{\theta}(\mathbf{x}, y)=\sum_{k=\zeta_{N}^{\theta}}^{N}\left(\begin{array}{l}
N \\
k
\end{array}\right) W_{Q}^{k}(\mathbf{x}, y)\left[1-W_{Q}(\mathbf{x}, y)\right]^{N-k}
$$


where, for positive $\theta$,

$$
\zeta_{N}^{\theta} \stackrel{\text { def }}{=} \max \left(\left\lceil\frac{N}{2}(1-\theta)\right\rceil, 0\right) .
$$

Figure 2 shows the behavior of $W_{Q^{N}}^{\theta}$ as a function of $W_{Q}$. The inflexion point of $W_{Q^{N}}^{\theta}$, when $N>1$ and $\zeta_{N}^{\theta}>1$ occurs 1 at $W_{Q}=\xi_{N}^{\theta}$ where

$$
\xi_{N}^{\theta} \stackrel{\text { def }}{=} \frac{\zeta_{N}^{\theta}-1}{N-1} .
$$

Since $N$ is a odd number, $\xi_{N}^{\theta}=1 / 2$ when $\theta=0$.

Equation 1 was the key starting point of [9] and [6] to obtain a margin bound for the weighted majority vote $B_{Q}$. The next important step is to upper bound $R^{\theta}\left(G_{Q^{N}}\right)$. 9] achieved this task by upper bounding

$$
\operatorname{Pr}_{(\mathbf{x}, y) \sim D}(y g(\mathbf{x}) \leq \theta)
$$

uniformly for all $g \in \mathcal{H}^{N}$ in terms of their empirical risk at margin $\theta$. Unfortunately, in the case of a finite set $\mathcal{H}$ of base classifiers, this step introduces a term in $O(\sqrt{(N / m) \log |\mathcal{H}|})$ in their risk bound by the application of the union bound over the set of at most $|\mathcal{H}|^{N}$ uniformly weighted majority votes of $N$ classifiers taken from $\mathcal{H}$.

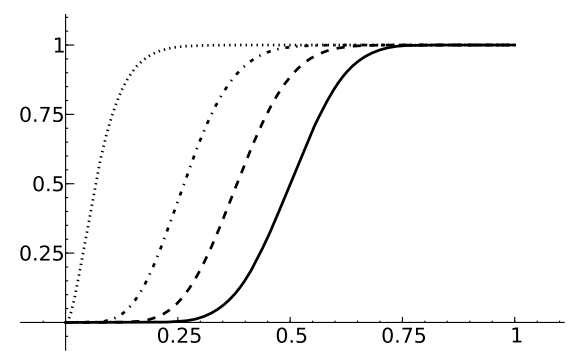

Fig. 2. Plots of $W_{Q^{N}}^{\theta}(\mathbf{x}, y)$ as a function of $W_{Q}(\mathbf{x}, y)$ for $N=25$ and $\theta=0,0.2,0.5$ and 0.9 (for curves from right to left respectively)

In contrast, [6] used the PAC-Bayes bound of [8] to upper bound $R^{\theta}\left(G_{Q^{N}}\right)$. This introduces a term in $O(\sqrt{(N / m) \mathrm{KL}(Q \| P)})$ in the risk bound and thus provides a significant improvement over the bound of 9 ] whenever $\operatorname{KL}(Q \| P) \ll$ $\ln |\mathcal{H}|$. Here we propose to obtain an even tighter bound by making use of Theorem 1 to upper bound $R^{\theta}\left(G_{Q^{N}}\right)$. This gives the following corollary.

${ }^{1} W_{Q^{N}}^{\theta}$ has no inflexion point when $N=1$ or $\zeta_{N}^{\theta} \leq 1$. 
Corollary 2. For any distribution $D$, any set $\mathcal{H}$ of base classifiers, any distribution $P$ of support $\mathcal{H}$, any $\delta \in(0,1]$, any $C>0$ and $\theta \geq 0$, and any integer $N>0$, we have

$$
\operatorname{Pr}_{S \sim D^{m}}\left(\begin{array}{rl}
\forall Q \text { on } \mathcal{H}: & \\
R\left(B_{Q}\right) \leq \frac{1}{1-e^{-C}} & \left\{1-\exp \left[-\left(C \cdot R_{S}^{\theta}\left(G_{Q^{N}}\right)\right.\right.\right. \\
& \left.\left.\left.+\frac{1}{m}\left[N \cdot \operatorname{KL}(Q \| P)+\ln \frac{1}{\delta}\right]\right)\right]\right\}+e^{-N \theta^{2} / 2}
\end{array}\right) \geq 1-\delta .
$$

To make this bound valid uniformly for any odd number $N$ and for any of the $(N+1) / 2+1$ values of $\theta$ mentioned before, the standard union bound argument tells us that it is sufficient to replace $\delta$ by $\frac{12}{\pi^{2}} \frac{1}{N^{2}(N+3)} \delta$ (in view of the fact that $\left.\sum_{i=1}^{\infty} i^{-2}=\pi^{2} / 6\right)$. Moreover, we should chose the value of $N$ to keep $e^{-N \theta^{2} / 2}$ comparable to the corresponding regularizer. This can be achieved by choosing

$$
N=\left\lceil\frac{2}{\theta^{2}} \ln \left(m\left[1-e^{-C}\right]\right)\right\rceil .
$$

Finally, it is important to mention that both [9] and [6] used

$$
R_{S}^{\theta}\left(G_{Q^{N}}\right) \leq R_{S}^{2 \theta}\left(B_{Q}\right)+e^{-N \theta^{2} / 2}
$$

to write their upper bound only in terms of the empirical risk $R_{S}^{2 \theta}\left(B_{Q}\right)$ at margin $2 \theta$ of the weighted majority vote $B_{Q}$, where

$$
R_{S}^{\theta}\left(B_{Q}\right) \stackrel{\text { def }}{=} \underset{(\mathbf{x}, y) \sim D}{\mathbf{E}} I(y \underset{h \sim Q}{\mathbf{E}} h(\mathbf{x}) \leq \theta) .
$$

This operation, however, contributes to an additional deterioration of the bound which, because of the presence of $R_{S}^{2 \theta}\left(B_{Q}\right)$, is now NP-hard to minimize. Consequently, for the purpose of bound minimization, it is preferable to work with a bound like Corollary 2 which depends on $R_{S}^{\theta}\left(G_{Q^{N}}\right)$ (and not on $R_{S}^{2 \theta}\left(B_{Q}\right)$ ).

\section{Proposed Learning Algorithms}

The task of the learning algorithm is to find the posterior $Q$ that minimizes the upper bound of Corollary 1 or Corollary 2 for fixed parameters $C, N$ and $\theta$. Note that for both of these cases, this is equivalent to find $Q$ that minimizes

$$
F(Q) \stackrel{\text { def }}{=} C \cdot R_{S}^{\theta}\left(G_{Q^{N}}\right)+\frac{1}{m} \cdot N \cdot \mathrm{KL}(Q \| P) .
$$

Indeed, minimizing $F(Q)$, when $\theta=0$, gives the posterior $Q$ minimizing the upper bound on $R\left(G_{Q^{N}}\right)$ given by Corollary 1 . Whereas minimizing $F(Q)$, when $\theta>0$, gives the posterior $Q$ minimizing the upper bound on $R\left(B_{Q}\right)$ given by Corollary 2 . 
Note that, for any fixed example $(\mathbf{x}, y), W_{Q^{N}}^{\theta}(\mathbf{x}, y)$ is a quasiconvex function of $Q$. However, a sum of quasiconvex functions is generally not quasiconvex and thus, not convex. Consequently, $R_{S}^{\theta}\left(G_{Q^{N}}\right)$, which is a sum of quasiconvex functions, is generally not convex with respect to $Q$. To obtain a convex optimization problem, we replace $R_{S}^{\theta}\left(G_{Q^{N}}\right)$ by the convex function $\mathcal{R}_{S}^{\theta}\left(G_{Q^{N}}\right)$ defined as

$$
\mathcal{R}_{S}^{\theta}\left(G_{Q^{N}}\right) \stackrel{\text { def }}{=} \frac{1}{m} \sum_{i=1}^{m} \mathcal{W}_{Q^{N}}^{\theta}\left(\mathbf{x}_{i}, y_{i}\right)
$$

where $\mathcal{W}_{Q^{N}}^{\theta}$ is the convex function of $W_{Q}$ which is the closest to $W_{Q^{N}}^{\theta}$ with the property that $\mathcal{W}_{Q^{N}}^{\theta}=W_{Q^{N}}^{\theta}$ when $W_{Q} \leq \xi_{N}^{\theta}$ (the inflexion point of $W_{Q^{N}}^{\theta}$ ). Hence,

$$
\mathcal{W}_{Q^{N}}^{\theta}(\mathbf{x}, y) \stackrel{\text { def }}{=}\left\{\begin{array}{l}
W_{Q^{N}}^{\theta}(\mathbf{x}, y) \quad \text { if } W_{Q}^{\theta}(\mathbf{x}, y) \leq \xi_{N}^{\theta} \\
\left.W_{Q^{N}}^{\theta}\right|_{W_{Q}=\xi_{N}^{\theta}}+\Delta_{N}^{\theta} \cdot\left(W_{Q}(\mathbf{x}, y)-\xi_{N}^{\theta}\right) \text { otherwise }
\end{array}\right.
$$

where $\Delta_{N}^{\theta}$ is the first derivative of $W_{Q^{N}}^{\theta}$ evaluated at its inflexion point $\xi_{N}^{\theta}$, i.e.,

$$
\left.\Delta_{N}^{\theta} \stackrel{\text { def }}{=} \frac{\partial W_{Q^{N}}^{\theta}}{\partial W_{Q}}\right|_{W_{Q}=\xi_{N}^{\theta}} .
$$

We thus propose to find $Q$ that minimizes 2

$$
\mathcal{F}(Q) \stackrel{\text { def }}{=} C \cdot \mathcal{R}_{S}^{\theta}\left(G_{Q^{N}}\right)+\frac{1}{m} \cdot N \cdot \mathrm{KL}(Q \| P) .
$$

We now restrict ourselves to the case where the set $\mathcal{H}$ of basis classifiers is finite. Let $\mathcal{H} \stackrel{\text { def }}{=}\left\{h_{1}, h_{2}, \ldots, h_{n}\right\}$. Given a distribution $Q$, let $Q_{i}$ be the weight assigned by $Q$ to classifier $h_{i}$.

For fixed $N, \mathcal{F}$ is a convex function of $Q$ with continuous first derivatives. Moreover, $\mathcal{F}$ is defined on a bounded convex domain which is the $n$-dimensional probability simplex for $Q$. Consequently, any local minimum of $\mathcal{F}$ is also a global minimum. Under these circumstances, coordinate descent minimization is guaranteed to converge to the global minimum of $\mathcal{F}$. To deal with the constraint that $Q$ is a distribution, we propose to perform the descent of $\mathcal{F}$ by using pairs of coordinates. For that purpose, let $Q_{\lambda}^{j, k}$ be the distribution obtained from $Q$ by transferring a weight $\lambda$ from classifier $h_{k}$ to classifier $h_{j}$ while keeping all other weights unchanged. Thus, for all $i \in\{1, \ldots, n\}$, we have

$$
\left(Q_{\lambda}^{j, k}\right)_{i} \stackrel{\text { def }}{=}\left\{\begin{array}{l}
Q_{i}+\lambda \text { if } i=j \\
Q_{i}-\lambda \text { if } i=k \\
Q_{i} \text { otherwise }
\end{array}\right.
$$

$\overline{2}$ Since $R_{S}^{\theta}\left(G_{Q^{N}}\right) \leq \mathcal{R}_{S}^{\theta}\left(G_{Q^{N}}\right)$, the bound of Corollary 2 holds whenever we replace $R_{S}^{\theta}\left(G_{Q^{N}}\right)$ by $\mathcal{R}_{S}^{\theta}\left(G_{Q^{N}}\right)$. 


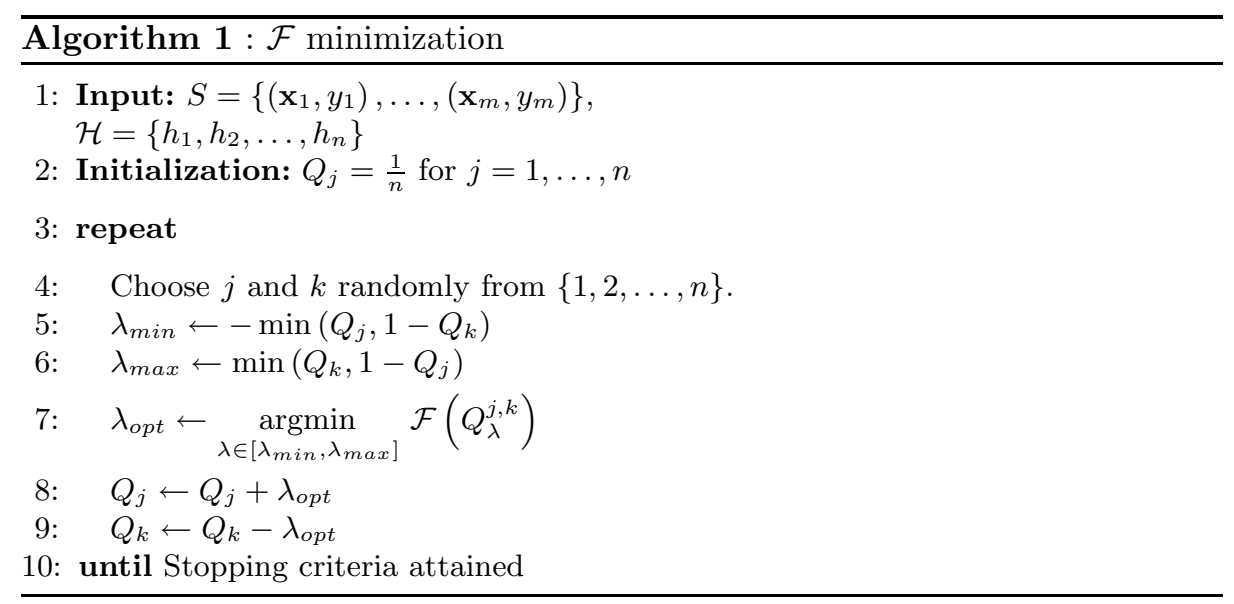

Note that in order for $Q_{\lambda}^{j, k}$ to remain a valid distribution, we need to choose $\lambda$ in the range $\left[-\min \left(Q_{j}, 1-Q_{k}\right), \min \left(Q_{k}, 1-Q_{j}\right)\right]$.

As described in Algorithm [1, each iteration of $\mathcal{F}$ minimization consists of the following two steps. We first choose $j$ and $k$ from $\{1,2, \ldots, n\}$ and then find $\lambda$ that minimizes $\mathcal{F}\left(Q_{\lambda}^{j, k}\right)$.

Since $\mathcal{F}$ is convex, the optimal value of $\lambda$ at each iteration is given by

$$
\frac{\partial \mathcal{F}\left(Q_{\lambda}^{j, k}\right)}{\partial \lambda}=C \frac{\partial \mathcal{R}_{S}^{\theta}\left(G_{\left(Q_{\lambda}^{j, k}\right)^{N}}\right)}{\partial \lambda}+\frac{1}{m} \cdot N \cdot \frac{\partial \mathrm{KL}\left(Q_{\lambda}^{j, k} \| P\right)}{\partial \lambda}=0 .
$$

For the uniform prior $\left(P_{i}=1 / n \forall i\right)$, we have

$$
\frac{\partial \mathrm{KL}\left(Q_{\lambda}^{j, k} \| P\right)}{\partial \lambda}=\ln \left(\frac{Q_{j}+\lambda}{Q_{k}-\lambda}\right) .
$$

Now, let $\mathcal{V}_{N}^{\theta}\left(W_{Q}\right) \stackrel{\text { def }}{=} \frac{\partial \mathcal{W}_{Q}^{\theta}}{\partial W_{Q}}$. We have

$$
\mathcal{V}_{N}^{\theta}\left(W_{Q}\right)= \begin{cases}\Delta_{N}^{\theta} \quad \text { if } W_{Q} \geq \xi_{N}^{\theta} & \\ \frac{N ! W_{Q}^{\zeta_{N}^{\theta}-1}\left(1-W_{Q}\right)^{N-\zeta_{N}^{\theta}}}{\left(\zeta_{N}^{\theta}-1\right) !\left(N-\zeta_{N}^{\theta}\right) !} & \text { otherwise }\end{cases}
$$

Then we have

$$
\frac{\partial \mathcal{R}_{S}^{\theta}\left(G_{\left(Q_{\lambda}^{j, k}\right)^{N}}\right)}{\partial \lambda}=\frac{1}{m} \sum_{i=1}^{m} \mathcal{V}_{N}^{\theta}\left(W_{Q_{\lambda}^{j, k}}\left(\mathbf{x}_{i}, y_{i}\right)\right) \frac{\partial W_{Q_{\lambda}^{j, k}}\left(\mathbf{x}_{i}, y_{i}\right)}{\partial \lambda} .
$$

From the definition of $W_{Q_{\lambda}^{j, k}}\left(\mathbf{x}_{i}, y_{i}\right)$, we find

$$
W_{Q_{\lambda}^{j, k}}\left(\mathbf{x}_{i}, y_{i}\right)=W_{Q}\left(\mathbf{x}_{i}, y_{i}\right)+\lambda \cdot D_{i}^{j, k},
$$


where

$$
D_{i}^{j, k} \stackrel{\text { def }}{=} I\left(h_{j}\left(\mathbf{x}_{i}\right) \neq h_{k}\left(\mathbf{x}_{i}\right)\right) y_{i} h_{k}\left(\mathbf{x}_{i}\right) .
$$

Hence, $\frac{\partial W_{Q_{\lambda}^{j, k}}\left(\mathbf{x}_{i}, y_{i}\right)}{\partial \lambda}=D_{i}^{j, k}$. Equation 5 therefore becomes

$$
\ln \left(\frac{Q_{j}+\lambda}{Q_{k}-\lambda}\right)+\frac{C}{N} \sum_{i=1}^{m} D_{i}^{j, k} \mathcal{V}_{N}^{\theta}\left(W_{Q_{\lambda}^{j, k}}\left(\mathbf{x}_{i}, y_{i}\right)\right)=0 .
$$

Since $\mathcal{V}_{N}^{\theta}$ is multiplied by $D_{i}^{j, k}$, we can replace in the above equation $W_{Q_{\lambda}^{j, k}}\left(\mathbf{x}_{i}, y_{i}\right)$ by $W_{Q}\left(\mathbf{x}_{i}, y_{i}\right)+\lambda y_{i} h_{k}\left(\mathbf{x}_{i}\right)$. If we now use $W_{Q}(i)$ as a shorthand notation for $W_{Q}\left(\mathbf{x}_{i}, y_{i}\right)$, Equation 5 finally becomes

$$
\ln \left(\frac{Q_{j}+\lambda}{Q_{k}-\lambda}\right)+\frac{C}{N} \sum_{i=1}^{m} D_{i}^{j, k} \mathcal{V}_{N}^{\theta}\left(W_{Q}(i)+\lambda y_{i} h_{k}\left(\mathbf{x}_{i}\right)\right)=0 .
$$

An iterative root-finding method, such as Newton's, can be used to solve Equation 6. Since we cannot factor out $\lambda$ from the summation in Equation 6 (as it can be done for AdaBoost), each iteration step of the root-finding method costs $\Theta(m)$ time. Therefore, Equation [6 is solved in $O(m k(\epsilon))$ time, where $k(\epsilon)$ denotes the number $k$ of iterations needed by the root-finding method to find $\lambda_{\text {opt }}$ within precision $\varepsilon$. Once we have found $\lambda_{\text {opt }}$, we update $Q$ with the new weights for $Q_{j}$ and $Q_{k}$ and update 3 each $W_{Q}(i)$ according to

$$
W_{Q}(i) \leftarrow W_{Q}(i)+\lambda D_{i}^{j, k} \quad \text { for } i \in\{1, \ldots, m\},
$$

in $\Theta(m)$ time. We repeat this process until all the weight modifications are within a desired precision $\varepsilon$.

Finally, if we go back to Equation 4 and consider the fact that $\operatorname{KL}(Q \| P) \leq$ $\ln |\mathcal{H}|$, we note $\mathcal{R}_{S}^{\theta}\left(G_{Q^{N}}\right)$ can dominate $N \cdot \mathrm{KL}(Q \| P)$. This is especially true whenever $S$ has the property that for any $Q$ there exist some training examples having $W_{Q}(\mathbf{x}, y)>1 / 2$. Indeed, in that case, the convexity of $\mathcal{W}_{Q}(\mathbf{x}, y)$ can force $\mathcal{R}_{S}^{\theta}\left(G_{Q^{N}}\right)$ to be always much larger than $N \cdot \operatorname{KL}(Q \| P)$ for any $Q$. In these circumstances, the posterior $Q$ that minimizes $\mathcal{R}_{S}^{\theta}\left(G_{Q^{N}}\right)$ should be similar to the one that minimizes $\mathcal{F}(Q)$. Consequently, we have also decided to minimize $\mathcal{R}_{S}^{\theta}\left(G_{Q^{N}}\right)$ at fixed $N$. In this case, we can drop the $C$ parameter and each iteration of the algorithm consists of solving Equation [6 without the presence of the logarithm term. Parameter $N$ then becomes the regularizer of the learning algorithm.

\section{Experimental Results}

We have tested our algorithms on more than 20 data sets. Except for MNIST, all data sets come from the UCI repository. Each data set was randomly split

${ }^{3}$ Initially we have $W_{Q}(i)=\frac{1}{n} \sum_{j=1}^{n} I\left(h_{j}\left(\mathbf{x}_{i}\right) \neq y_{i}\right)$ for $i \in\{1, \ldots, m\}$. 
Table 1. Results for Algorithm $1 \mathcal{F}$ minimization, at zero margin $(\theta=0)$

\begin{tabular}{|l||c|c|c|c|c||c|c|c|c|c|}
\hline \multicolumn{1}{|l||}{ Dataset } & \multicolumn{4}{|c||}{ Bound } & \multicolumn{5}{c|}{ CV - R(B $\left.\mathbf{B}_{\mathbf{Q}}\right)$} \\
\hline Name & $R\left(B_{Q}\right)$ & $R\left(G_{Q^{N}}\right)$ & $N$ & $C$ & Bnd & $R\left(B_{Q}\right)$ & $R\left(G_{Q^{N}}\right)$ & $N$ & $C$ & Bnd \\
\hline Adult & 0.206 & 0.206 & 1 & 0.2 & 0.245 & 0.152 & 0.171 & 499 & 20 & 0.958 \\
\hline Letter:AB & 0.093 & 0.092 & 1 & 0.5 & 0.152 & 0.009 & 0.043 & 49 & 2 & 0.165 \\
\hline Letter:DO & 0.141 & 0.143 & 1 & 0.5 & 0.199 & 0.027 & 0.040 & 999 & 50 & 0.808 \\
\hline Letter:OQ & 0.257 & 0.257 & 1 & 0.5 & 0.313 & 0.041 & 0.052 & 4999 & 200 & 0.994 \\
\hline MNIST:0vs8 & 0.046 & 0.054 & 1 & 1 & 0.102 & 0.007 & 0.015 & 49 & 50 & 0.415 \\
\hline MNIST:1vs7 & 0.045 & 0.058 & 1 & 1 & 0.115 & 0.011 & 0.017 & 49999 & 100 & 0.506 \\
\hline MNIST:1vs8 & 0.042 & 0.108 & 25 & 1 & 0.233 & 0.021 & 0.030 & 499 & 500 & 0.835 \\
\hline MNIST:2vs3 & 0.138 & 0.159 & 1 & 0.5 & 0.215 & 0.045 & 0.066 & 75 & 20 & 0.600 \\
\hline Mushroom & 0.019 & 0.035 & 49 & 1 & 0.097 & 0.000 & 0.001 & 999 & 100 & 0.317 \\
\hline Ringnorm & 0.046 & 0.117 & 999999 & 1 & 0.252 & 0.026 & 0.034 & 9999 & 200 & 0.998 \\
\hline Waveform & 0.083 & 0.117 & 25 & 0.5 & 0.172 & 0.081 & 0.114 & 49 & 0.5 & 0.172 \\
\hline
\end{tabular}

into a training set $S$ of $|S|$ examples and a testing set $T$ of $|T|$ examples. The number $d$ of attributes for each data set is also specified in Table 2.

For all tested algorithms, we have used decision stumps for the set $\mathcal{H}$ of base classifiers. Each decision stump $h_{\ell, t, b}$ is a threshold classifier that outputs $+b$ if the $\ell$ th attribute of the input example exceeds a threshold value $t$, and $-b$ otherwise, where $b \in\{-1,+1\}$. For each attribute, at most ten equally spaced possible values for $t$ were determined a priori.

The results for the first set of experiments are summarized in Table 1. For these experiments, we have minimized the objective function $\mathcal{F}$ at zero margin as described by Algorithm 1 and have compared two different ways of choosing the hyperparameters $N$ and $C$ of $\mathcal{F}$. For the Bound method, the values chosen for $N$ and $C$ were those minimizing the risk bound given by Corollary 1 on $S$ whereas, for the $\mathbf{C V}-\mathbf{R}\left(\mathbf{B}_{\mathbf{Q}}\right)$ method, the values of these hyperparameters were those minimizing the 10-fold cross-validation score (on $S$ ) of the weighted majority vote $B_{Q}$. For all cases, $R\left(B_{Q}\right)$ and $R\left(G_{Q^{N}}\right)$ refer, respectively, to the empirical risk of the weighted majority vote $B_{Q}$ and of the randomized majority vote $G_{Q^{N}}$ computed on the testing set $T$. Also indicated, are the values found for $N$ and $C$. In all cases, $N$ was chosen among a set 17 values between 1 and $10^{6}-1$ and $C$ was chosen among a set 5 of 15 values between 0.02 and 1000 .

As we can see in Table 1 the bound values are indeed much smaller when $N$ and $C$ are chosen such as to minimize the risk bound. However, both the weighted majority vote $B_{Q}$ and the randomized majority vote $G_{Q^{N}}$ obtained in this manner performed much worse than those obtained when $C$ and $N$ were

\footnotetext{
${ }^{4}$ Values for $N$ :

$\{1,3,5,7,9,25,49,75,99,499,999,4999,9999,49999,99999,499999,999999\}$.

${ }^{5}$ Values for $C$ :

$\{0.02,0.05,0.1,0.2,0.5,1,2,5,10,20,50,100,200,500,1000\}$.
} 
Table 2. Results for Algorithm $1, \mathcal{F}$ minimization, compared with AdaBoost (AB)

\begin{tabular}{|l|c|c|c||c||c|c|c||c|c|c|c|}
\hline \multicolumn{4}{|c||}{ Dataset } & \multicolumn{1}{c||}{ AB } & \multicolumn{3}{c||}{ Algo 1, $\theta=\mathbf{0}$} & \multicolumn{3}{c|}{ Algo 1, $>>\mathbf{0}$} \\
\hline Name & $|S|$ & $|T|$ & $d$ & $R\left(B_{Q}\right)$ & $R\left(B_{Q}\right)$ & $N$ & $C$ & $R\left(B_{Q}\right)$ & $N$ & $C$ & $\theta$ \\
\hline Adult & 1809 & 10000 & 14 & $\mathbf{0 . 1 4 9}$ & 0.152 & 499 & 20 & 0.153 & 49999 & 1000 & 0.017 \\
\hline BreastCancer & 343 & 340 & 9 & 0.053 & 0.041 & 7 & 1 & $\mathbf{0 . 0 3 8}$ & 499 & 1000 & 0.153 \\
\hline Credit-A & 353 & 300 & 15 & 0.170 & $\mathbf{0 . 1 5 0}$ & 9999 & 2 & $\mathbf{0 . 1 5 0}$ & 49999 & 5 & 0.015 \\
\hline Glass & 107 & 107 & 9 & 0.178 & $\mathbf{0 . 1 3 1}$ & 49 & 500 & $\mathbf{0 . 1 3 1}$ & 499 & 200 & 0.137 \\
\hline Haberman & 144 & 150 & 3 & $\mathbf{0 . 2 6 0}$ & 0.273 & 1 & 0.001 & 0.273 & 5 & 0.02 & 0.647 \\
\hline Heart & 150 & 147 & 13 & 0.252 & 0.177 & 75 & 1 & $\mathbf{0 . 1 7 0}$ & 4999 & 5 & 0.045 \\
\hline Ionosphere & 176 & 175 & 34 & 0.120 & $\mathbf{0 . 1 0 3}$ & 499 & 200 & 0.114 & 4999 & 200 & 0.045 \\
\hline Letter:AB & 500 & 1055 & 16 & 0.010 & 0.009 & 49 & 2 & $\mathbf{0 . 0 0 6}$ & 4999 & 10 & 0.050 \\
\hline Letter:DO & 500 & 1058 & 16 & 0.036 & $\mathbf{0 . 0 2 7}$ & 999 & 50 & 0.032 & 999 & 50 & 0.112 \\
\hline Letter:OQ & 500 & 1036 & 16 & $\mathbf{0 . 0 3 8}$ & 0.041 & 4999 & 200 & 0.044 & 49999 & 20 & 0.016 \\
\hline Liver & 170 & 175 & 6 & 0.320 & 0.349 & 25 & 2 & $\mathbf{0 . 3 1 4}$ & 999 & 20 & 0.101 \\
\hline MNIST:0vs8 & 500 & 1916 & 784 & 0.008 & $\mathbf{0 . 0 0 7}$ & 49 & 50 & $\mathbf{0 . 0 0 7}$ & 499 & 50 & 0.158 \\
\hline MNIST:1vs7 & 500 & 1922 & 784 & 0.013 & $\mathbf{0 . 0 1 1}$ & 49999 & 100 & 0.013 & 9999 & 50 & 0.035 \\
\hline MNIST:1vs8 & 500 & 1936 & 784 & 0.025 & 0.021 & 499 & 500 & $\mathbf{0 . 0 2 0}$ & 999 & 50 & 0.112 \\
\hline MNIST:2vs3 & 500 & 1905 & 784 & 0.047 & 0.045 & 75 & 20 & $\mathbf{0 . 0 3 4}$ & 4999 & 20 & 0.050 \\
\hline Mushroom & 4062 & 4062 & 22 & $\mathbf{0 . 0 0 0}$ & $\mathbf{0 . 0 0 0}$ & 999 & 100 & $\mathbf{0 . 0 0 0}$ & 4999 & 200 & 0.058 \\
\hline Ringnorm & 3700 & 3700 & 20 & 0.043 & $\mathbf{0 . 0 2 6}$ & 9999 & 200 & 0.028 & 49999 & 500 & 0.018 \\
\hline Sonar & 104 & 104 & 60 & 0.231 & $\mathbf{0 . 1 9 2}$ & 25 & 20 & 0.231 & 999 & 500 & 0.096 \\
\hline Usvotes & 235 & 200 & 16 & $\mathbf{0 . 0 5 5}$ & $\mathbf{0 . 0 5 5}$ & 1 & 0.2 & $\mathbf{0 . 0 5 5}$ & 25 & 1 & 0.633 \\
\hline Waveform & 4000 & 4000 & 21 & 0.085 & $\mathbf{0 . 0 8 1}$ & 49 & 0.5 & $\mathbf{0 . 0 8 1}$ & 999 & 100 & 0.129 \\
\hline Wdbc & 285 & 284 & 30 & 0.049 & $\mathbf{0 . 0 3 5}$ & 499 & 20 & 0.039 & 9999 & 100 & 0.034 \\
\hline
\end{tabular}

selected by cross-validation. The difference is statistically significant 6 in every cases except on the Waveform data set. We can also observe that the values of $N$ chosen by cross-validation are much larger than those selected by the risk bound. When $N$ is large, the stochastic predictor $G_{Q^{N}}$ becomes close to the deterministic weighted majority vote $B_{Q}$ but we can still observe an overall superiority for the $B_{Q}$ predictor.

The results for the second set of experiments are summarized in Table 2 where we also provide a comparison to AdaBoost7. The hyperparameters $C$ and $N$ for these experiments were selected based on the 10-fold cross-validation score (on $S$ ) of $B_{Q}$. We have also compared the results for Algorithm 1 when $\theta$ is fixed to zero and when $\theta$ can take non-zero values. The results presented here for Algorithm 1] at non-zero margin are those when $\theta$ is fixed to the value given by Equation 2. Interestingly, as indicated in Table 3. we have found that fixing $\theta$ in this way gave, overall, equivalent performance as choosing it by cross-validation (the difference is never statistically significant).

\footnotetext{
${ }^{6}$ To determine whether or not a difference of empirical risk measured on the testing set $T$ is statistically significant, we have used the test set bound method of [5] (based on the binomial tail inversion) with a confidence level of $95 \%$.

${ }^{7}$ For these experiments, the number of boosting rounds was fixed to 200 .
} 
Table 3. Comparison of results when $\theta$ is chosen by 10 -fold cross-validation to those when $\theta$ is fixed to the value given by Equation 2

\begin{tabular}{|l|c||c|c|c|c||c|c|c|c|}
\hline Dataset & \multicolumn{1}{|c||}{ AB } & \multicolumn{3}{c||}{ Algo 1, CV- $\theta$} & \multicolumn{3}{c|}{ Algo 1, $\theta^{*}$} \\
\hline Name & $R\left(B_{Q}\right)$ & $R\left(B_{Q}\right)$ & $N$ & $C$ & $\theta$ & $R\left(B_{Q}\right)$ & $N$ & $C$ & $\theta$ \\
\hline Adult & $\mathbf{0 . 1 4 9}$ & 0.152 & 499 & 20 & 0.005 & 0.153 & 49999 & 1000 & 0.017 \\
\hline BreastCancer & 0.053 & 0.041 & 25 & 10 & 0.05 & $\mathbf{0 . 0 3 8}$ & 499 & 1000 & 0.153 \\
\hline Credit-A & 0.170 & $\mathbf{0 . 1 5 0}$ & 9999 & 2 & 0 & $\mathbf{0 . 1 5 0}$ & 49999 & 5 & 0.015 \\
\hline Glass & 0.178 & $\mathbf{0 . 1 3 1}$ & 9999 & 200 & 0.05 & $\mathbf{0 . 1 3 1}$ & 499 & 200 & 0.137 \\
\hline Haberman & 0.260 & $\mathbf{0 . 2 5 3}$ & 3 & 500 & 0.5 & 0.273 & 5 & 0.02 & 0.647 \\
\hline Heart & 0.252 & 0.177 & 49999 & 5 & 0.025 & $\mathbf{0 . 1 7 0}$ & 4999 & 5 & 0.045 \\
\hline Ionosphere & 0.120 & $\mathbf{0 . 1 1 4}$ & 49999 & 1000 & 0.1 & $\mathbf{0 . 1 1 4}$ & 4999 & 200 & 0.045 \\
\hline Letter:AB & 0.010 & $\mathbf{0 . 0 0 6}$ & 4999 & 10 & 0.025 & $\mathbf{0 . 0 0 6}$ & 4999 & 10 & 0.050 \\
\hline Letter:DO & 0.036 & $\mathbf{0 . 0 2 9}$ & 499 & 10 & 0.025 & 0.032 & 999 & 50 & 0.112 \\
\hline Letter:OQ & $\mathbf{0 . 0 3 8}$ & 0.041 & 999 & 100 & 0.005 & 0.044 & 49999 & 20 & 0.016 \\
\hline Liver & 0.320 & 0.349 & 25 & 2 & 0 & $\mathbf{0 . 3 1 4}$ & 999 & 20 & 0.101 \\
\hline MNIST:0vs8 & 0.008 & $\mathbf{0 . 0 0 7}$ & 99 & 1000 & 0.1 & $\mathbf{0 . 0 0 7}$ & 499 & 50 & 0.158 \\
\hline MNIST:1vs7 & 0.013 & $\mathbf{0 . 0 1 2}$ & 4999 & 50 & 0.025 & 0.013 & 9999 & 50 & 0.035 \\
\hline MNIST:1vs8 & 0.025 & 0.021 & 499 & 500 & 0 & $\mathbf{0 . 0 2 0}$ & 999 & 50 & 0.112 \\
\hline MNIST:2vs3 & 0.047 & 0.049 & 99 & 100 & 0.1 & $\mathbf{0 . 0 3 4}$ & 4999 & 20 & 0.050 \\
\hline Mushroom & $\mathbf{0 . 0 0 0}$ & $\mathbf{0 . 0 0 0}$ & 999 & 100 & 0 & $\mathbf{0 . 0 0 0}$ & 4999 & 200 & 0.058 \\
\hline Ringnorm & 0.043 & $\mathbf{0 . 0 2 7}$ & 9999 & 200 & 0.005 & 0.028 & 49999 & 500 & 0.018 \\
\hline Sonar & 0.231 & $\mathbf{0 . 1 4 4}$ & 49 & 500 & 0.025 & 0.231 & 999 & 500 & 0.096 \\
\hline Usvotes & $\mathbf{0 . 0 5 5}$ & $\mathbf{0 . 0 5 5}$ & 1 & 0.2 & 0 & $\mathbf{0 . 0 5 5}$ & 25 & 1 & 0.633 \\
\hline Waveform & 0.085 & $\mathbf{0 . 0 8 1}$ & 49 & 0.5 & 0 & $\mathbf{0 . 0 8 1}$ & 999 & 100 & 0.129 \\
\hline Wdbc & 0.049 & $\mathbf{0 . 0 3 5}$ & 499 & 20 & 0 & 0.039 & 9999 & 100 & 0.034 \\
\hline
\end{tabular}

Going back to Table 2, we see that the results for Algorithm 1 when $\theta>0$ are competitive (but different) with those obtained at $\theta=0$. There is thus no competitive advantage at choosing a non-zero margin value (but there is no disadvantage either and no computational disadvantage since the value of $\theta$ is not chosen by cross-validation). Finally, the results indicate that both of these algorithms perform generally better than AdaBoost but the results are significant only on the Ringnorm data set.

As described in the previous section, we have also minimized $\mathcal{R}_{S}^{\theta}\left(G_{Q^{N}}\right)$ at a fixed number of voters $N$, which now becomes the regularizer of the learning algorithm. This algorithm has the significant practical advantage of not having an hyperparameter $C$ to tune. Three versions of this algorithm are compared in Table 4. In the first version, $\mathcal{R}_{S}^{\theta}\left(G_{Q^{N}}\right)$-min, the value of $\theta$ was selected based on the 10-fold cross-validation score (on $S$ ) of $B_{Q}$. In the second version, the value of $\theta$ was fixed to $\sqrt{(1 / N) \ln (2 m)}$. In the third version, the value of $\theta$ was fixed to zero. We see, in Table 4, that all three versions are competitive to one another. The difference in the results was never statistically significant. Hence, again, there is no competitive advantage at choosing a non-zero margin value for the empirical risk of the randomized majority vote. We also find that results for all three versions are competitive with AdaBoost. The difference was significant 
Table 4. Results for the Algorithm that minimizes $\mathcal{R}_{S}^{\theta}\left(G_{Q^{N}}\right)$

\begin{tabular}{|l||c||c|c|c||c|c|c||c|c|}
\hline \multicolumn{1}{|l||}{ Dataset } & \multicolumn{1}{|c||}{$\mathbf{A B}$} & \multicolumn{2}{|c||}{$\mathcal{R}_{\mathbf{S}}^{\theta}\left(\mathbf{G}_{\left.\mathbf{Q}^{\mathbf{N}}\right)}\right) \mathbf{m i n}}$. & \multicolumn{2}{c||}{$\mathcal{R}_{\mathbf{S}}^{\theta}\left(\mathbf{G}_{\mathbf{Q}^{\mathbf{N}}}\right), \theta^{*}$} & \multicolumn{2}{c|}{$\mathcal{R}_{\mathbf{S}}^{\mathbf{0}}\left(\mathbf{G}_{\mathbf{Q}^{\mathbf{N}}}\right)$} \\
\hline Name & $R\left(B_{Q}\right)$ & $R\left(B_{Q}\right)$ & $N$ & $\theta$ & $R\left(B_{Q}\right)$ & $N$ & $\theta$ & $R\left(B_{Q}\right)$ & $N$ \\
\hline Adult & $\mathbf{0 . 1 4 9}$ & 0.153 & 999 & 0.091 & 0.153 & 999 & 0.091 & 0.151 & 75 \\
\hline BreastCancer & 0.053 & $\mathbf{0 . 0 4 4}$ & 499 & 0.114 & $\mathbf{0 . 0 4 4}$ & 499 & 0.114 & $\mathbf{0 . 0 4 4}$ & 25 \\
\hline Credit-A & 0.170 & $\mathbf{0 . 1 3 3}$ & 25 & 0.512 & $\mathbf{0 . 1 3 3}$ & 25 & 0.512 & 0.137 & 9 \\
\hline Glass & 0.178 & $\mathbf{0 . 1 3 1}$ & 499 & 0.104 & $\mathbf{0 . 1 3 1}$ & 499 & 0.104 & $\mathbf{0 . 1 3 1}$ & 49 \\
\hline Haberman & $\mathbf{0 . 2 6 0}$ & 0.273 & 7 & 0.899 & 0.273 & 7 & 0.899 & 0.273 & 1 \\
\hline Heart & 0.252 & 0.190 & 499 & 0.107 & 0.190 & 499 & 0.107 & $\mathbf{0 . 1 7 7}$ & 49 \\
\hline Ionosphere & $\mathbf{0 . 1 2 0}$ & 0.131 & 4999 & 0.034 & 0.131 & 4999 & 0.034 & 0.143 & 999 \\
\hline Letter:AB & 0.010 & $\mathbf{0 . 0 0 1}$ & 99999 & 0.008 & $\mathbf{0 . 0 0 1}$ & 99999 & 0.008 & 0.006 & 99999 \\
\hline Letter:DO & 0.036 & $\mathbf{0 . 0 2 6}$ & 49999 & 0.012 & $\mathbf{0 . 0 2 6}$ & 49999 & 0.012 & 0.028 & 49999 \\
\hline Letter:OQ & $\mathbf{0 . 0 3 8}$ & 0.043 & 4999 & 0.037 & 0.043 & 4999 & 0.037 & 0.048 & 999 \\
\hline Liver & $\mathbf{0 . 3 2 0}$ & 0.343 & 999 & 0.076 & 0.343 & 999 & 0.076 & 0.349 & 49999 \\
\hline MNIST:0vs8 & 0.008 & 0.008 & 4999 & 0.037 & 0.008 & 4999 & 0.037 & $\mathbf{0 . 0 0 7}$ & 75 \\
\hline MNIST:1vs7 & 0.013 & 0.011 & 99999 & 0.008 & 0.011 & 99999 & 0.008 & $\mathbf{0 . 0 1 0}$ & 49999 \\
\hline MNIST:1vs8 & 0.025 & 0.020 & 4999 & 0.037 & 0.020 & 4999 & 0.037 & $\mathbf{0 . 0 1 8}$ & 4999 \\
\hline MNIST:2vs3 & 0.047 & 0.041 & 4999 & 0.037 & 0.041 & 4999 & 0.037 & $\mathbf{0 . 0 3 5}$ & 49999 \\
\hline Mushroom & $\mathbf{0 . 0 0 0}$ & $\mathbf{0 . 0 0 0}$ & 4999 & 0.042 & $\mathbf{0 . 0 0 0}$ & 4999 & 0.042 & $\mathbf{0 . 0 0 0}$ & 999 \\
\hline Ringnorm & 0.043 & $\mathbf{0 . 0 2 8}$ & 49999 & 0.013 & $\mathbf{0 . 0 2 8}$ & 49999 & 0.013 & 0.029 & 9999 \\
\hline Sonar & 0.231 & 0.212 & 4999 & 0.033 & 0.212 & 4999 & 0.033 & $\mathbf{0 . 1 9 2}$ & 99 \\
\hline Usvotes & $\mathbf{0 . 0 5 5}$ & $\mathbf{0 . 0 5 5}$ & 25 & 0.496 & $\mathbf{0 . 0 5 5}$ & 25 & 0.496 & $\mathbf{0 . 0 5 5}$ & 1 \\
\hline Waveform & 0.085 & $\mathbf{0 . 0 8 0}$ & 499 & 0.134 & $\mathbf{0 . 0 8 0}$ & 499 & 0.134 & 0.081 & 99 \\
\hline Wdbc & 0.049 & 0.039 & 9999 & 0.025 & 0.039 & 9999 & 0.025 & $\mathbf{0 . 0 3 5}$ & 75 \\
\hline
\end{tabular}

on the Ringnorm and Letter:AB data sets (in favor of $\mathcal{R}_{S}^{\theta}\left(G_{Q^{N}}\right)$ minimization). Hence, $\mathcal{R}_{S}^{\theta}\left(G_{Q^{N}}\right)$ minimization at a fixed number $N$ of voters appears to be a good substitute to regularized variants of boosting.

\section{Conclusion}

In comparison with other state-of-the-art learning strategies such as boosting, our numerical experiments indicate that learning by probing the empirical risk of the randomized majority vote is an excellent strategy for producing weighted majority votes that generalize well. We have shown that this learning strategy is strongly supported by PAC-Bayes theory because the proposed risk bound immediately gives the objective function to minimize. However, the precise weighting of the KL regularizer versus the empirical risk that appears in the bound is not the one giving the best generalization. In practice, substantially less weighting should be given to the regularizer. In fact, we have seen that minimizing the empirical risk of the randomized majority vote at a fixed number of voters, without considering explicitly the KL regularizer, gives equally good results. Among the different algorithms that we have proposed, the latter appears to be the best substitute to regularized variants of boosting because the number of voters is the only hyperparameter to tune. 
We have also found that probing the empirical risk of the randomized majority vote at zero margin gives equally good weighted majority votes as those produced by probing the empirical risk at finite margin.

\section{Acknowledgments}

Work supported by NSERC discovery grants 122405 and 262067.

\section{References}

1. Catoni, O.: PAC-Bayesian supervised classification: the thermodynamics of statistical learning. Monograph series of the Institute of Mathematical Statistics (December 2007), http://arxiv.org/abs/0712.0248

2. Freund, Y., Schapire, R.E.: A decision-theoretic generalization of on-line learning and an application to boosting. Journal of Computer and System Sciences 55, 119-139 (1997)

3. Germain, P., Lacasse, A., Laviolette, F., Marchand, M.: PAC-Bayesian Learning of Linear Classifiers. In: Bottou, L., Littman, M. (eds.) Proceedings of the 26th International Conference on Machine Learning (ICML 2009), pp. 353-360. Omnipress, Montreal (June 2009)

4. Jaakkola, T., Meila, M., Jebara, T.: Maximum entropy discrimination. In: Advances in neural information processing systems, vol. 12. MIT Press, Cambridge (2000)

5. Langford, J.: Tutorial on practical prediction theory for classification. Journal of Machine Learning Research 6, 273-306 (2005)

6. Langford, J., Seeger, M., Megiddo, N.: An improved predictive accuracy bound for averaging classifiers. In: Brodley, C.E., Danyluk, A.P. (eds.) Proceedings of the Eighteenth International Conference on Machine Learning (ICML 2001), June 28-July 1, pp. 290-297. Morgan Kaufmann, San Francisco (2001)

7. McAllester, D.: PAC-Bayesian stochastic model selection. Machine Learning 51, 5-21 (2003)

8. McAllester, D.A.: PAC-Bayesian model averaging. In: COLT, pp. 164-170 (1999)

9. Schapire, R.E., Freund, Y., Bartlett, P., Lee, W.S.: Boosting the margin: A new explanation for the effectiveness of voting methods. The Annals of Statistics 26, 1651-1686 (1998)

10. Seeger, M.: PAC-Bayesian generalization bounds for gaussian processes. Journal of Machine Learning Research 3, 233-269 (2002) 